\title{
Spin dynamics triggered by subterahertz magnetic field pulses
}

Zhao Wang, Matthäus Pietz, Jakob Walowski, Arno Förster, Mihail I. Lepsa, and Markus Münzenberg

Citation: Journal of Applied Physics 103, 123905 (2008);

View online: https://doi.org/10.1063/1.2940734

View Table of Contents: http://aip.scitation.org/toc/jap/103/12

Published by the American Institute of Physics

\section{Articles you may be interested in}

Perspective: Ultrafast magnetism and $\mathrm{THz}$ spintronics

Journal of Applied Physics 120, 140901 (2016); 10.1063/1.4958846

Ultrabroadband single-cycle terahertz pulses with peak fields of $300 \mathrm{kV} \mathrm{cm}^{-1}$ from a metallic spintronic emitter Applied Physics Letters 110, 252402 (2017); 10.1063/1.4986755

Antiferromagnetic resonance excitation by terahertz magnetic field resonantly enhanced with split ring resonator Applied Physics Letters 105, 022410 (2014); 10.1063/1.4890475

Low frequency terahertz-induced demagnetization in ferromagnetic nickel

Applied Physics Letters 108, 182903 (2016); 10.1063/1.4948472

Narrow-band tunable terahertz emission from ferrimagnetic $\mathrm{Mn}_{3-\mathrm{x}} \mathrm{Ga}$ thin films

Applied Physics Letters 109, 032403 (2016); 10.1063/1.4958855

Coherent terahertz emission from ferromagnetic films excited by femtosecond laser pulses

Applied Physics Letters 84, 3465 (2004); 10.1063/1.1737467

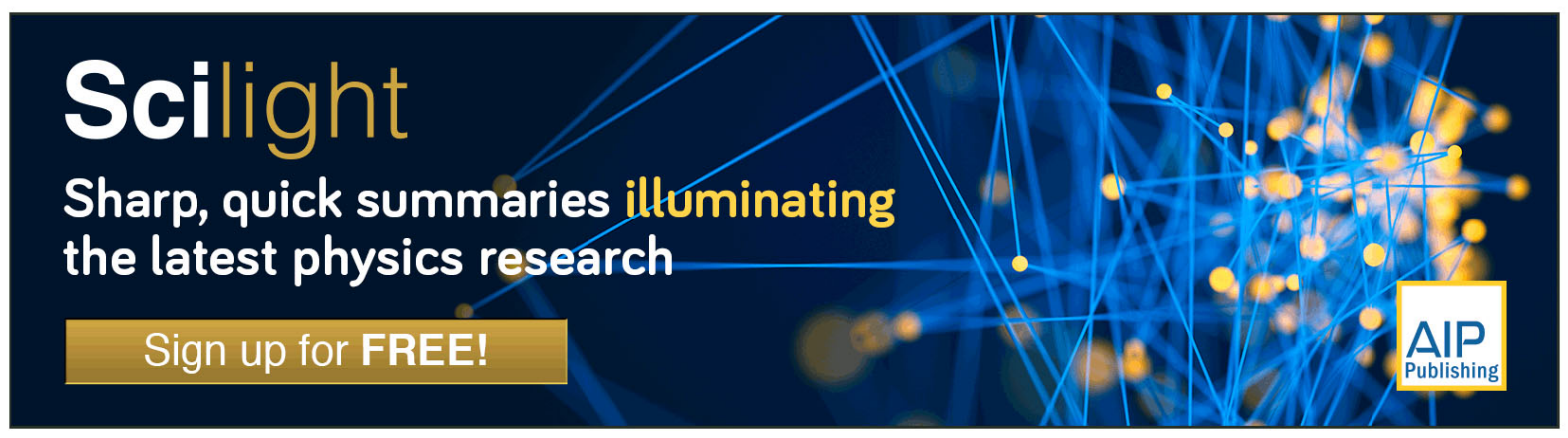




\title{
Spin dynamics triggered by subterahertz magnetic field pulses
}

\author{
Zhao Wang, ${ }^{1}$ Matthäus Pietz, ${ }^{1}$ Jakob Walowski, ${ }^{1}$ Arno Förster, ${ }^{2}$ Mihail I. Lepsa, ${ }^{3}$ and \\ Markus Münzenberg ${ }^{1, a)}$ \\ ${ }^{1}$ IV. Physikalisches Institut, Georg-August-Universität, Göttingen 37077, Germany \\ ${ }^{2}$ Fachhochschule Aachen, Jülich 52428, Germany \\ ${ }^{3}$ Institute für Bio- und Nanosysteme (IBN-1), Forschungszentrum Jülich GmbH, Jülich 52425, Germany
}

(Received 18 November 2007; accepted 14 April 2008; published online 18 June 2008)

\begin{abstract}
Current pulses of up to $20 \mathrm{~A}$ and as short as $3 \mathrm{ps}$ are generated by a low-temperature-grown GaAs photoconductive switch and guided through a coplanar waveguide, resulting in a $0.6 \mathrm{~T}$ subterahertz magnetic field pulse. The pulse length is directly calibrated using photocurrent autocorrelation. Magnetic excitations in Fe microstructures are studied by time-resolved Kerr spectroscopy. An ultrafast response time (within less than 10 ps of the magnetization) to the subterahertz electromagnetic field pulse is shown. (C) 2008 American Institute of Physics.
\end{abstract}

[DOI: 10.1063/1.2940734]

\section{INTRODUCTION}

There are different ways to drive magnetization dynamics to the limits: In the time domain, the most prominent are femtosecond all-optical excitations ${ }^{1-3}$ and field pulse excitations. ${ }^{4-7}$ In the first case the time scales are extremely short (approximately picoseconds), ${ }^{8}$ but the direction of the excitation cannot be controlled. In general, the ultrafast perturbation by the femtosecond laser pulse generates a broad spectrum of excitations from high energy (high $k$-vector) to low energy modes of the coherent precession $(k=0){ }^{9}$

Øersted field pulses are generally limited to field strength (approximately a few milliteslas) and temporal resolution ( $>30$ ps) since they are restricted to the capabilities of high frequency electronics. The record is held by an alternative cost-intensive approach: The generation of a magnetic field pulse by relativistic electron bunches. At the Stanford Linear Accelerator Center (SLAC), the magnetic field yields up to more than $5 \mathrm{~T}$ in amplitude and less than a picosecond in pulse length. ${ }^{10,11}$ From the load of the ultrafast and strong field pulses, a fracture of the magnetization is observable. Tudosa et al. ${ }^{11}$ therefore postulate a limit for the fastest switching of a recording media determined by the magnetization breakup and driven by the intrinsic nonlinearity of the Landau-Lifshitz-Gilbert equation. Random thermal fluctuations, always present in the magnetic system, are amplified by the driving field pulse. However, to make an electronic device spin ultrafast, a field of about $10 \mathrm{~T}$ is needed in order to switch the magnetization within a picosecond.

In the following, we shall present an on-chip geometry approach, which uses optical switches, as a source of picosecond and high-power current pulses to drive the magnetization dynamics toward a similar value range. The transient magnetic field is generated by a photoconductive (or Auston) switch $^{12}$ and the magnetization dynamics are probed with a delay by a probe pulse via the magneto-optic Kerr effect (MOKE), as shown in Fig. 1(b). The method, named

\footnotetext{
${ }^{a)}$ Author to whom correspondence should be addressed. Electronic mail: mmuenze@gwdg.de.
}

magneto-optic sampling, has been intensively developed by M. R. Freeman over the last few years $[4,13]$ and allows the observation of the magnetization transient directly in time. Because of the ultrashort carrier lifetime, low-temperaturegrown GaAs (LT-GaAs) is of special interest for applications up to terahertz bandwidths and is widely used. ${ }^{14}$ Here we connect both techniques, magneto-optic sampling and terahertz pulse generation, to establish a SLAC on-chip. The process is as follows: First the terahertz-current pulse is characterized by a photocurrent autocorrelation technique. Then the magnetic response of an Fe stripe to the subterahertz field pulse, experimentally determined by magneto-optic sampling, is given.

\section{EXPERIMENT}

In the following, the preparation details and dimensions of the on-chip devices are given. The photoconductive switches are prepared by optical lithography on a $1 \mu \mathrm{m}$ thick LT-GaAs film grown by molecular beam epitaxy on a semi-insulating GaAs wafer at $200{ }^{\circ} \mathrm{C}$ and annealed at

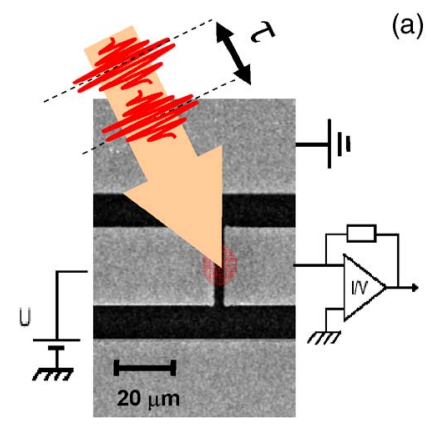

(a)

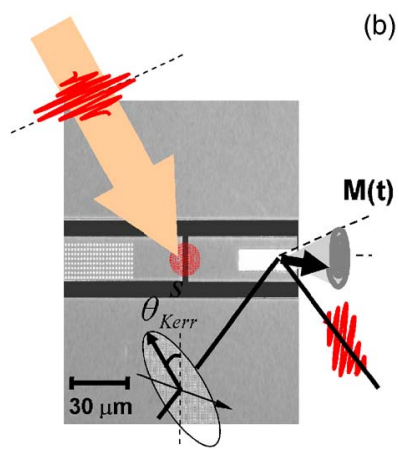

FIG. 1. (Color online) (a) Scanning electron microscope image of the optical switch with schematic representation of the photocurrent autocorrelation experiment to determine the current pulse characteristics of the LT-GaAs photo switch. (b) Optical microscope image of the optical switch area including the patterned magnetic Fe structures at both sides: an array of two micron-sized structures to the left and an Fe stripe pattern to the right. On top, the schematic representation of the experiment to monitor the magnetization dynamics is given. 
$600{ }^{\circ} \mathrm{C}$ for $10 \mathrm{~min}$ inside the chamber in As-rich conditions. ${ }^{15}$ Characterization of the photocarrier lifetimes by time-resolved reflectivity measurements reveal two dominating relaxation times of the carriers of 70 and $140 \mathrm{fs}$, respectively. In the next step, by using optical lithography, a $22.5 \mu \mathrm{m}$ wide center conductive strip (5 nm Ti/ $30 \mathrm{~nm} \mathrm{Al}$ ) with a gap of $3 \mu \mathrm{m}$ is evaporated onto the LT-GaAs substrate. Figure 1(a) shows a scanning electron microscope image of the metal-semiconductor-metal (MSM) gap and its dimensions. In addition the experimental geometry is given schematically for the photocurrent autocorrelation experiment. Two pulses delayed by a time $\tau$ illuminate the $3 \mu \mathrm{m}$ MSM gap and the photocurrent is determined. The electrical pulses are generated by the femtosecond laser illumination of the MSM gap and then transmitted through the coplanar waveguide [Fig. 1(b)]. When passing the coplanar waveguide, an ultrafast magnetic field pulse is generated with a dominating in-plane component in the middle of the center conductor.

To complete the magneto-optic sampling device, the magnetic structures are patterned directly on top of the terahertz waveguide close to the MSM gap (on-chip geometry) as seen in the optical microscope image shown in Fig. 1(b): A $5 \mathrm{~nm} \mathrm{MgO/} 30 \mathrm{~nm}$ Fe film is evaporated on the center conductor and structured using electron-beam lithography by a lift-off process. The Fe structure similar to the one discussed in Sec. III B can be seen in Fig. 1(b), $20 \mu \mathrm{m}$ $\times 100 \mu \mathrm{m}$ in size, close to the MSM gap. On top of the microscopy image, a schematic drawing of the magnetooptic sampling experiment is given. While the first pulse still illuminates the MSM gap, the second pulse is reflected at the magnetic structure. Via the MOKE, the magnetization dynamics are determined at a delay time $\tau$ using a double modulation technique. ${ }^{16}$ Since the skin depth for an Fe film is $3.5 \mathrm{~nm}$ at $1 \mathrm{THz}$ frequency only, a considerable current flow through the $\mathrm{Fe}$ film itself has to be avoided by the insertion of a thin insulating $5 \mathrm{~nm} \mathrm{MgO}$ layer. The laser system used for the carrier excitation is a Ti:sapphire oscillator with a RegA amplifier that generates 60 fs pulses $(\sim 1 \mu \mathrm{J})$ with a central wavelength of $800 \mathrm{~nm}$ and a repetition rate of $f_{\text {rep }}=250 \mathrm{kHz}$.

\section{EXPERIMENTAL RESULTS}

\section{A. Current pulse characteristics}

The advantage of the photocurrent autocorrelation technique presented here is that as opposed to other techniques (e.g., picosecond electro-optic ${ }^{17}$ or photoconductive sampling using a dual photoconductor circuit) ${ }^{18}$ the same sample geometry as for the magneto-optic sampling can be used to characterize the electric pulse length. Only a single photoconductor is needed for the photocurrent autocorrelation measurement. A prerequisite is that the photocurrent increases nonlinearly with the rise of laser power as seen in Fig. 2(a): At a constant voltage, the photocurrent saturates for high fluence. Because of the high defect density of the LT-GaAs film, the MSM contact has Ohmic-like characteristics. $^{15,19}$ It has been shown in Jacobsen et al. ${ }^{20}$ that from the photocurrent autocorrelation experiments, the time-
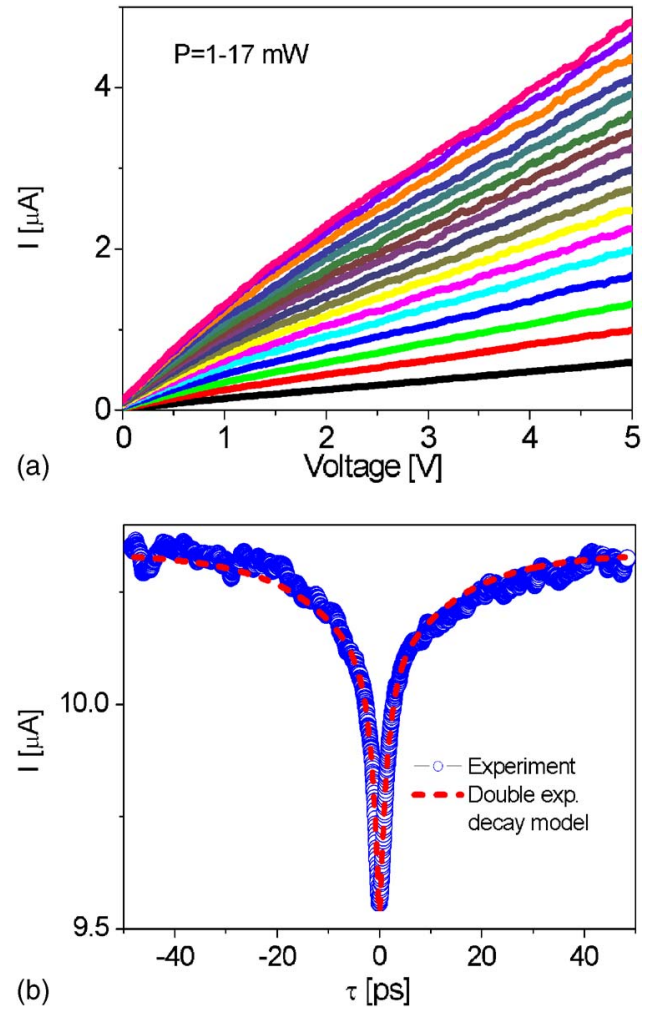

FIG. 2. (Color online) (a) Current vs voltage characteristics of the photoswitch structure ( $3 \mu \mathrm{m}$ gap) under illumination varying the laser power from 1 to $17 \mathrm{~mW}$, showing the nonlinearity of the photocurrent with the illumination power. (b) Photocurrent autocorrelation ( $3 \mu \mathrm{m}$ gap structure, 3 $\mathrm{V}$ gap voltage). The solid line shows the analysis using a double exponential decay of the photocurrent toward zero delay $\tau$ between the two laser pulses illuminating the gap.

dependent carrier density can be extracted. Therefore the photocurrent autocorrelation curve can be analyzed using an exponential decay function where the time constants are related to carrier relaxation times. In the following we allow two relaxation times $\left(\tau_{\mathrm{el}}\right.$ and $\left.\tau_{\text {geom }}\right)$ to describe the experimental data; then the photocurrent as a function of the delay time $\tau$ between the laser pulses is given by

$$
I(\tau)=I_{0}-I_{\mathrm{el}} e^{-|\tau| / \tau_{\mathrm{el}}}-I_{\mathrm{geom}} e^{-|\tau| \tau_{\mathrm{geom}}},
$$

where $I_{0}$ is the maximum photocurrent [Fig. 2(b)]. Parameter sets $I_{e l}, \tau_{\text {el }}$ and $I_{\text {geom }}, \tau_{\text {geom }}$ characterize the electrical pulse decay. It is found that the first relaxation time of $\tau_{\mathrm{el}}$ $=1-1.5 \mathrm{ps}$ is related to the carrier recombination time. The ratio of the current amplitudes is about $I_{\mathrm{el}}: I_{\text {geom }}>1.5: 1$. For a finger-switch geometry where the gap region is curved in order to increase the optically active area, the second, slower decay $\left(\tau_{\text {geom }}=5-25 \mathrm{ps}\right.$, dependent on the alignment) can be suppressed. Therefore from the geometry dependence we conclude that antenna effects of the metallization interacting with the femtosecond-light pulse are responsible for the second, slower contribution. ${ }^{21}$ The average pulse length for the $3 \mu \mathrm{m}$ gap switch geometry extracted from the photocurrent autocorrelation experiments is therefore estimated to be $\bar{\tau}$ $=3 \pm 1$ ps from the autocorrelation experiment. This value is in good agreement with the results from photoconductive sampling experiments using a second photoconductive 
switch as it was determined earlier. ${ }^{18}$ An estimate of the maximum current is given by

$$
I_{\mathrm{max}}=\frac{I_{\mathrm{av}}-I_{\mathrm{dark}}}{f_{\text {rep }} \bar{\tau}} .
$$

It can be easily seen that a high resistance of the nonilluminated switch is needed to suppress the dark current. The average current is up to $16 \mu \mathrm{A}$ for an $80 \mathrm{~V}$ bias voltage and $6 \mathrm{~mW}$ average laser power $(250 \mathrm{kHz}$ repetition rate) and results in a current amplitude of $I_{\max }=20 \pm 8$ A. Assuming a homogeneous current distribution throughout the coplanar waveguide (the skin depth of the center conductor materials at $1 \mathrm{THz}$ is about $100 \mathrm{~nm}$ ), the numerical calculations of the magnetic field distribution above the center conductor result in a homogeneous field component $\left(B_{y}\right)$ parallel to the surface of the center conductor of $B_{\max }=0.6 \pm 2 \mathrm{~T}$. Compared with prior, standard approaches which commonly use Auston switches or electrical pulsers for magneto-optic sampling and synchrotron-based experiments (and which are becoming increasingly important as a novel tool to image magnetization dynamics), this is a significant increase in magnetic field strength. The out-of-plane field component $\left(B_{z}\right)$ has a strong contribution at the edges of the conductor, with opposite sign, but it is zero at the center and will be neglected in the following.

\section{B. Magnetization dynamics}

For the magneto-optic sampling experiments as depicted in Fig. 1(b), the experimental geometry and a schematic diagram of the sample in the on-chip geometry are shown. An external magnetic static field of $0.03 \mathrm{~T}$ is applied along the $20 \mu \mathrm{m} \times 100 \mu \mathrm{m} 30 \mathrm{~nm}$ Fe film structure to saturate the film along that direction. The Fe film senses the magnetic near field of the terahertz pulse propagating through the center conductor that is directed perpendicular to the static magnetic field. The evolution of the time-resolved Kerr rotation $\Delta \theta_{\mathrm{Kerr}}(\tau)$ is shown in Fig. 3. $\Delta \theta_{\mathrm{Kerr}}(\tau)$ is probed for 0 (reference), 28 and $60 \mathrm{~V}$ voltage applied to the gap. This voltage corresponds to about $0.20 \pm 8$ and $0.40 \pm 16 \mathrm{~T}$. As a reference a Gaussian function with 3 ps width at half maximum is shown. A steep rising edge of the differential Kerr signal $\Delta \theta_{\text {Kerr }}(\tau)$ well below $10 \mathrm{ps}$ is found. This response time does not depend significantly on the field pulse strength. Only the amplitude is about doubled as a result of doubling the field strength. For the reference experiment with zero voltage applied across the photoconductive switch, the observed differential Kerr signal $\Delta \theta_{\text {Kerr }}(\tau)$ is zero and thus excludes a direct demagnetization by the laser pump pulse. Micromagnetic simulations using OOMMF (Ref. 22) represented by the dashed lines are overlaid on the experimental data. For the micromagnetic simulations, an Fe structure of $5 \times 15 \mu \mathrm{m}^{2}$ in size and $30 \mathrm{~nm}$ in thickness and a cell size of $d_{\text {cell }} \leq 50 \mathrm{~nm}$ were used. The results were tested for convergence for smaller cell sizes. Surprisingly we find a good agreement with the experimental data without adjusting any parameters (dashed lines in Fig. 3). In the inset of Fig. 3, the Kerr signal shown for the time scale of up to 500 ps (gap voltage of 28 V) reveals a critically damped oscillation. The high damping

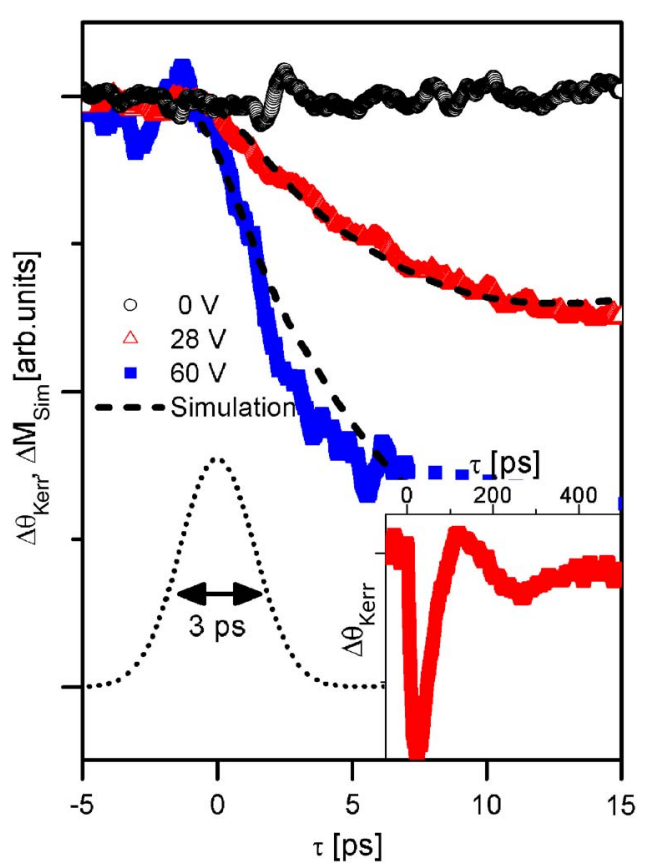

FIG. 3. (Color online) Magnetic response of a $30 \mathrm{~nm}$ thick Fe stripe pattern on the center conductor for the short time scale for different voltages, 0 (reference), 28, and $60 \mathrm{~V}$, applied to the photoconductive switch. Overlaid on the data, the results of the micromagnetic simulation are shown (dashed line). In the inset, the signal for $28 \mathrm{~V}$ is shown on a larger time scale. As a reference a Gaussian function ( $3 \mathrm{ps}$ ) is plotted to indicate the field pulse (dotted line).

found in response to the terahertz pulse indicates an increase in the apparent damping. This may be interpreted as a signature of the broad spectrum of spin-wave excitations leading to a strong decay of the signal in total. Increasing the field pulse strength can lead to increased damping, as shown in previous cases; the activation of additional damping channels is actually a strongly debated field ${ }^{23-26}$ to which we hope to contribute through determining the results of driving the field pulse strength even higher. Details of this increase have yet to be verified in further experiments. The major aim in further experiments will be to realize a full $180^{\circ}$ switching of the magnetization of the Fe film within one magnetic ultrashort pulse. This will be possible in future photoconductive switch devices approaching $10 \mathrm{~T}$ field amplitude.

\section{CONCLUSIONS}

Applying high voltages up to $80 \mathrm{~V}$ and an average laser power of $10 \mathrm{~mW}$, the devices are driven to the limit of their stability in the present design. Also low probe beam intensities limit the sensitivity of the Kerr signal detection. However, we have shown that it is possible to generate $0.6 \pm 2 \mathrm{~T}$, $3 \pm 1$ ps long magnetic field pulses and to study the magnetization dynamics excited by a subterahertz electromagnetic field pulse on a chip. The response time of the magnetic signal is found to be within the order of $10 \mathrm{ps}$, as expected from micromagnetic calculations. An improved switch design using a finger-switch structure with a larger gap area will stabilize the photoconductive switch and allow pulse strengths of a few teslas (similar to the SLAC experiments, but without using a linear accelerator or synchrotron) in an 
on-chip experiment with comparatively simple laboratory environment in the future. We expect to study similar effects to these, driving the terahertz radiation emission in ultrafast demagnetization experiments ${ }^{27}$ using devices with pulse-rise times below the picosecond range in the future.

\section{ACKNOWLEDGMENTS}

Support by the Deutsche Forschungsgemeinschaft within the priority program SPP 1133 is gratefully acknowledged.

${ }^{1}$ E. Beaurepaire, J.-C. Merle, A. Daunois, and J.-Y. Bigot, Phys. Rev. Lett. 76, 4250 (1996).

${ }^{2}$ M. van Kampen, C. Jozsa, J. T. Kohlhepp, P. LeClair, L. Lagae, W. J. M. de Jonge, and B. Koopmans, Phys. Rev. Lett. 88, 227201 (2002).

${ }^{3}$ G. Ju, A. Vertikov, A. V. Nurmikko, C. Canady, G. Xiao, R. F. C. Farrow, and A. Cebollada, Phys. Rev. B 57, R700 (1998).

${ }^{4}$ W. K. Hiebert, A. Stankiewitz, and M. R. Freeman Phys. Rev. Lett. 79, 1134 (1997); B. C. Choi, J. Ho, G. Arnup, and M. R. Freeman, ibid. 95, 237211 (2005).

${ }^{5}$ T. Gerrits, H. A. M. van der Berg, J. Hohlfeld, L. Bär, and T. Rasing, Nature (London) 418, 509 (2002).

${ }^{6}$ M. Bauer, R. Lopusnik, J. Fassbender, and B. Hillebrands, Appl. Phys. Lett. 76, 2758 (2000).

${ }^{7}$ H. W. Schumacher, C. Chappert, R. C. Sousa, P. P. Freitas, and J. Miltat, Phys. Rev. Lett. 90, 017204 (2003).

${ }^{8}$ C. Jozsa, J. H. H. Rietjens, M. van Kampen, E. Smalbrugge, M. K. Smit, W. J. M. de Jonge, and B. Koopmans, J. Appl. Phys. 95, 7447 (2004).

${ }^{9}$ M. Djordjevic and M. Münzenberg, Phys. Rev. B 75, 012404 (2007).

${ }^{10}$ C. H. Back, D. Weller, J. Heidmann, D. Mauri, D. Guarisco, E. L. Garwin, and H. C. Siegmann, Phys. Rev. Lett. 81, 3251 (1998).

${ }^{11}$ I. Tudosa, C. Stamm, A. B. Kashuba, F. King, H. C. Siegmann, J. Stöhr, G. Ju, B. Lu, and D. Weller, Nature (London) 428, 831 (2004).

${ }^{12}$ D. H. Auston, Appl. Phys. Lett. 26, 101 (1975); D. H. Auston, K. P.
Cheung, and R. P. Smith, ibid. 45, 284 (1984).

${ }^{13}$ M. R. Freeman, M. J. Brady, and J. Smyth, Appl. Phys. Lett. 60, 2555 (1992); A. Y. Elezzabi and M. R. Freeman, ibid. 68, 3546 (1996).

${ }^{14}$ M. C. Beard, G. M. Turner, and C. A. Schmuttenmaer, J. Phys. Chem. B 106, 7146 (2002).

${ }^{15}$ P. Kordoš, A. Förster, M. Marso, and F. Rüders, Electron. Lett. 34, 119 (1998); P. Kordoš, M. Marso, A. Förster, J. Darmo, J. Betko, and G. Nimtz, Appl. Phys. Lett. 71, 1118 (1997).

${ }^{16}$ M. Djordjevic, G. Eilers, A. Parge, M. Münzenberg, and J. S. Moodera, J. Appl. Phys. 99, 08F308 (2006).

${ }^{17}$ J. A. Valdmanis, G. A. Mourou, and C. W. Gabel, Appl. Phys. Lett. 41, 211 (1982).

${ }^{18}$ N. Zamdmer, Q. Hu, S. Vergehse, and A. Förster, Appl. Phys. Lett. 74, 1039 (1999).

${ }^{19}$ R. Adam, M. Mikulics, A. Förster, J. Schelten, M. Siegel, P. Kordos, X. Zheng, S. Wu, and R. Sobolewski, Appl. Phys. Lett. 81, 3485 (2002).

${ }^{20}$ R. H. Jacobsen, K. Birkelund, T. Holst, P. Uhd Jepsen, and S. R. Keiding, J. Appl. Phys. 79, 2649 (1996).

${ }^{21}$ Generally to suppress the antenna effects, the polarization of pump and probe beam is in crossed geometry and rotated $\pm 45^{\circ}$ with respect to the metallization edge.

${ }^{22}$ M. J. Donahue and D. G. Porter, "OOMMF User's Guide, Version 1.0," National Institute of Standards and Technology, Interagency Report No. NISTIR 6376, 1999.

${ }^{23}$ R. D. McMichael, 52nd Annual Conference on Magnetism and Magnetic Materials, Tampa, FL, 6 November 2007 (unpublished).

${ }^{24}$ H. Suhl, J. Phys. Chem. Solids 1, 209 (1957).

${ }^{25}$ M. L. Schneider, Th. Gerrits, A. B. Kos, and T. J. Silva J. Appl. Phys. 102, 053910 (2007); Th. Gerrits, P. Krivosik, M. L. Schneider, C. E. Patton, and T. J. Silva, Phys. Rev. Lett. 98, 207602 (2007).

${ }^{26}$ G. Müller, M. Münzenberg, G.-X. Miao, and A. Gupta, Phys. Rev. B 77, 020412(R) (2008).

${ }^{27}$ E. Beaurepaire, G. M. Turner, S. M. Harrel, M. C. Beard, J.-Y. Bigot, and C. A. Schmuttenmaer, Appl. Phys. Lett. 84, 3465 (2004); S. M. Harrel, J. M. Schleicher, E. Beaurepaire, J.-Y. Bigot, and C. A. Schmuttenmaer, Proc. SPIE 5929, 592910 (2005). 